\title{
151. Chromosome Condition of an Intersex Swine ${ }^{1,2)}$
}

\author{
By Sajiro Makino,*) Masao S. SASaki,*) Toshio Sofuni** \\ and Tsune IsHIKAWA**) \\ (Comm. by Yoshimaro TANAKA, M.J.A., Nov. 12, 1962)
}

It is indeed within only the recent several years that the significance of aberrant chromosome constitution associated with human subjects with congenital syndromes and abnormal sexual development has been definitely established following advances in cytological techniques available for reliable preparations from surgical or marrow biopsies as well as from leucocytes in short-term culture. During the period 1959 to 1962, over 600 papers have been published in the field of human cytogenetics regarding significant associations between abnormal chromosome conditions and various disease states. It is quite natural that advances in cytological techniques and the increase of knowledge in human cytogenetics excites one's desire to investigate chromosome conditions of domestic mammals in relation to clinical defects and sexual abnormalities, since such a project has remained largely unexplored in mammals. In a former paper, Makino et al. (1962) reported the chromosomes of bovine freemartins. In the present paper is presented a preliminary description of the chromosome condition in an intersex swine.

Clinical features: The propositus was a Yorkshire breed (Sus scrofa), aged 7 months. Masculine characteristics were found in its face and skeleton. Opening of the urethra was of female type. Clitoris was unusually enlarged and had a penile configuration. It was found by operation that this specimen had female-type internal genitalia, consisting of apparently normal vagina and bipartite uterus. A pair of gonads on both sides possessed an appearance of intraabdominal testes (Fig. 1), with a structure resembling the epididymis. Neither prostate nor evidence of seminal vesicles was seen.

Histological structure: Histologically both left and right testes were hypoplastic in structure. There were extremely atrophic seminiferous tubules as generally found in undescended testes. The tubules contained an unusually large number of sustentacular cells. There were in the tubules a very few germ-cells in poor develop-

1) Contribution No. 569 from the Zoological Institute, Faculty of Science, Hokkaido University, Sapporo.

2) Supported by a grant-in-aid for Fundamental Scientific Research from the Ministry of Education (No. 0438, 1962).

*) Zoological Institute, Hokkaido University, Sapporo.

**) Department of Veterinary Obstetrics, Hokkaido University, Sapporo. 
ment without evidence of spermatogenesis. Well-developed interstitial cells occurred with an extreme increase in both number and quality. Hyperplasia of the interstitial cells was more remarkable in the right testis than in the left one (Fig. 2). The hyperplastic interstitial cells contained a considerable number of granules; it is sufficient to suggest their high activity of secreting function. Microscopical examinations of the epididymis revealed a rather normal structure of epididymal tubules, though no spermatozoa were found (Fig. 3). It is then evident that there is no structure which corresponds to the ovarian element in this gonad.

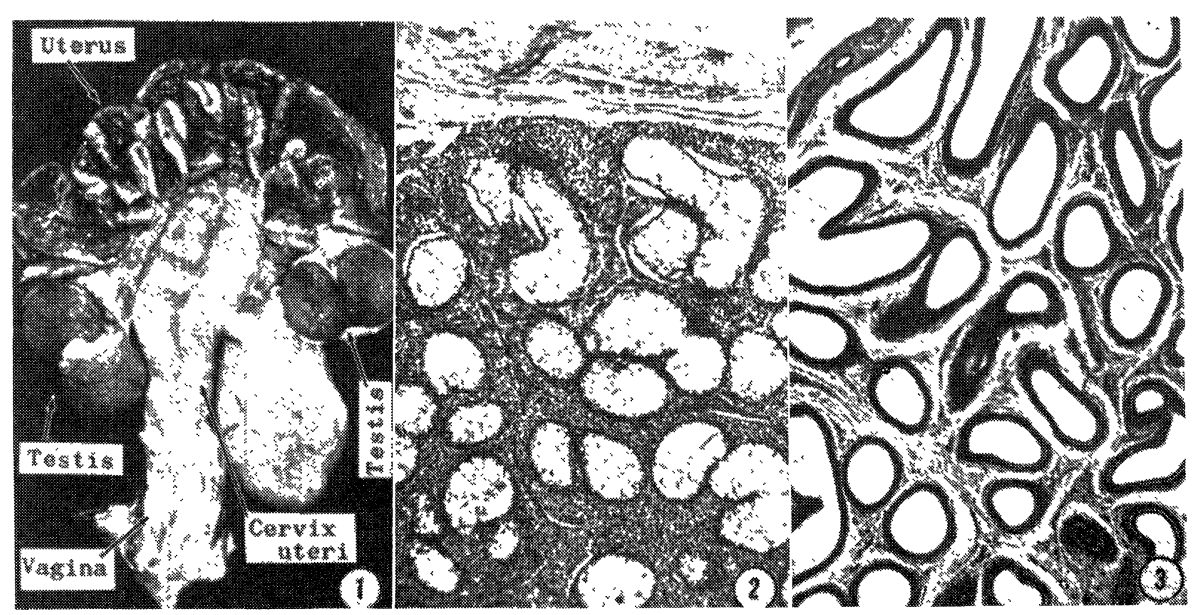

Fig. 1. Internal genitalia of an intersex swine. Apparently normally developed uterus, cervix uteri and vagina with intra-abdominal testes.

Fig. 2. Microscopical structure of right testis of an intersex swine $(\times 60)$. $(\times 60)$.

Fig. 3. Microscopical structure of the epididymis of the left testis

Cytological findings: A blood specimen was used for culture of leucocytes in order to observe the chromosomes. The culture procedure followed the method of Hungerford et al. (1959) with some modifications: heparinized blood was placed in an ice bath for about 2 hours, and then the resultant supernate was cultivated. Culture was set up as a mixture of the supernate and culture medium in a proportion of 1:3 (Makino et al. 1962). After 2 days' incubation at $37^{\circ} \mathrm{C}$, colchicine treatment $\left(50 \times 10^{-8} \mathrm{M}\right.$, for 1.5 to 2 hours $)$ was administrated in order to arrest the cells at metaphase. The cells were decanted from the culture bottles by means of trypsin digestion and collected by centrifugal sedimentation (1000 rpm, for 5 minutes). They were squashed according to the water pretreatment, acetic dahlia squash method. Further, somatic chromosomes of 
normal swine, male and female, were examined with the same procedure as above.

The diploid number of chromosomes in somatic cells in culture was determined to be 38 in both sexes of normal specimens, which consisted of 36 autosomes and a pair of sex chromosomes, two X's in female cells, and an $\mathrm{X}$ and a $\mathrm{Y}$ in male cells (Figs. 4-5), The $\mathrm{X}$ chromosome was one of the medium sized submetacentric elements, while the $\mathrm{Y}$ chromosome was the smallest metacentric element; it was easily distinguishable from the others. ${ }^{1)}$

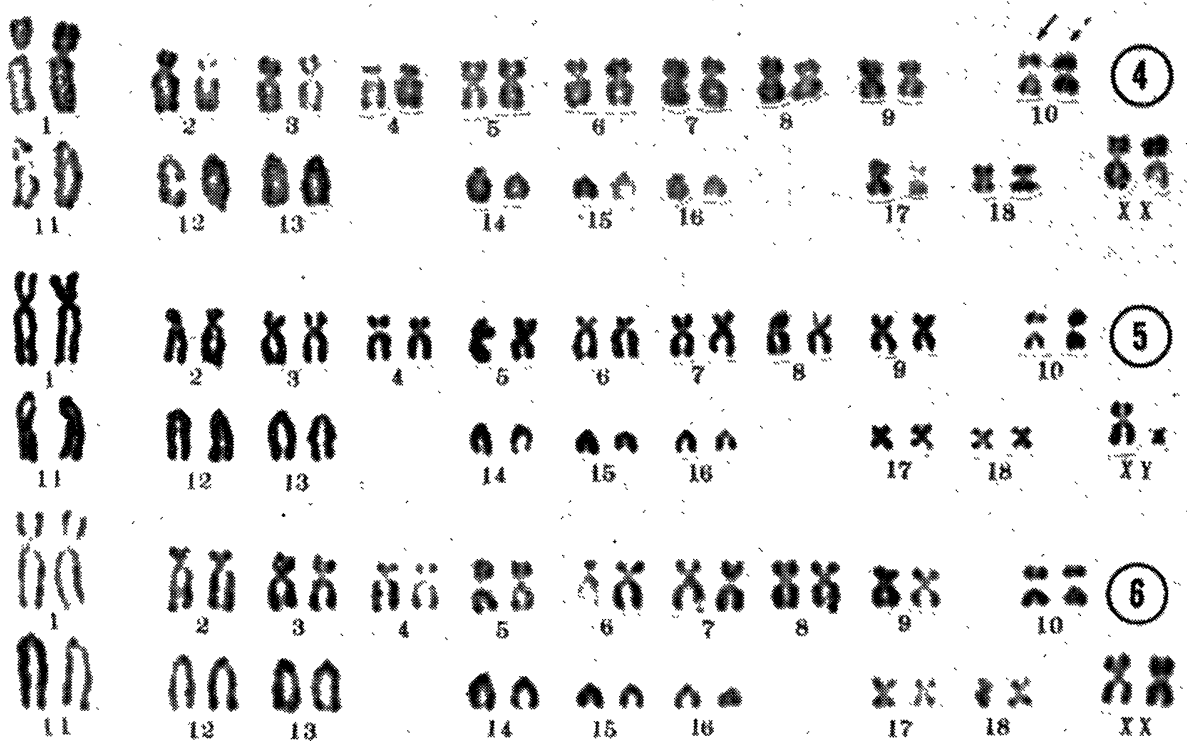

Fig. 4-5. Karyotype analyses of the somatic chromosomes of the normal pigs (leukocyte cultures). Fig. 4: normal female complement, Fig. 5: normal male complement.

Fig. 6. The same of an intersex swine (leucocyte culture), showing an apparently normal female complement.

A total of 40 metaphasic cells from an intersex swine rendered a reliable count of chromosomes: 36 cells out of them showed a normal diploid complement consisting of 38 chromosomes. Chromosome analyses made on five cells with 38 chromosomes revealed that they had in each an apparently normal female complex, comprising 36 autosomes and a pair of $\mathrm{X}$ chromosomes (Fig. 6).

Remarks: In this paper, the chromosomes of an intersex swine were studied in comparison with the normal somatic chromosomes of the pig.

1) The chromosome number of the pig as reported by one of the present authors in an earsly paper (Makino, S. 1944, Cytologia 13:170-178) is hereby revised. 
To date, there have been reported some eight cases of human hermaphrodites which show a remarkable testicular development but have an apparently normal female constitution of chromosomes: they were categorized to true hermaphrodites having both testis and ovary, or ovotestes (Hungerford et al. 1959, Harnden and Armstrong 1959, Sasaki and Makino 1960, Ferguson-Smith et al. 1960, de Assis et al. 1960, Gordon et al. 1960). It is a matter of problem that the development of testicular elements occurs in patients or in animals without a demonstrable $\mathrm{Y}$ chromosome. In addition, Bloise et al. (1960) and Atkins and Engel (1962) reported an XO sex-mechanism in patients with rudimentary or prepubertal uterus and embryonic testes. The intersex swine here considered had bilateral intraabdominal testes and an apparently normal female chromosome constitution. No ovarian structure was detected. The features do not correspond to those in the human hermaphrodites as mentioned above. The intersexuality of this animal may be interpreted thus: the male gonads acquired masculinization although the genetic sex has retained a female constitution.

In conclusion, the intersex swine with testicular development showed an apparently normal female constitution of chromosomes. Critical incision remains to be performed in order to make further extensive investigation on the occurrence of male gonads in this animal.

\section{References}

Atkins, L. and Engel, E. (1962): Lancet, ii, 20-23.

Bloise, W., de Assis, L. M., Bottura, C. and Ferrari, I. (1960): Lancet, ii, 1059-1060. De Assis, L. M., Epps, D. R. and Bottura, C. (1960): Lancet, ii, 129-130.

Ferguson-Smith, M. A., Johnston, A. W. and Weinberg, A. N. (1960): Lancet, i, 126128.

Gordon, R. R., O'Gorman, F. J. P., Dewhurst, C. J., and Blank, C. E. (1960): Lancet, ii, $736-739$.

Harnden, D. G. and Armstrong, C. N. (1959): Brit. Med. Jour., 2, 1287-1288.

Hungerford, D. A., Donnelly, A. J., Nowell, P. C. and Beck, S. (1959): Amer. J. Human Genet., 11, 215-236.

Makino, S., Kikuchi, Y., Sasaki, M. S., Sasaki, M. and Yoshida, M. (1962): Chromosoma (Berl.), 13, 148-162.

Sasaki, M. and Makino, S. (1960): Texas Rep. Biol. Med., 18, 493-500. 